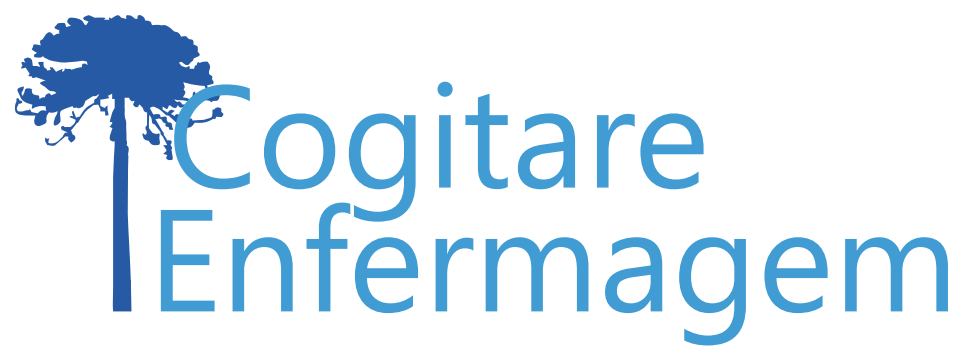

\title{
ANÁLISE ESPACIAL DO ESTUPRO EM ADOLESCENTES: CARACTERÍSTICAS E IMPACTOS
}

Paula Daniella de Abreu1, Zailde Carvalho dos Santos², Firley Poliana da Silva Lúcio ${ }^{3}$, Tarcisio Neves da Cunha4, Ednaldo Cavalcante de Araújo ${ }^{5}$, Claudia Benedita dos Santos 6 , Eliane Maria Ribeiro de Vasconcelos ${ }^{7}$

\section{RESUMO}

Objetivo: analisar a correlação entre as taxas brutas de violência por estupro e ano de ocorrência e o padrão espacial da ocorrência dos casos notificados de violência por estupro contra adolescentes. Método: estudo ecológico com tendência temporal, constituído a partir das notificações no período de 2009 a 2014, referentes aos casos de violência por estupro contra adolescentes residentes no estado de Pernambuco, coletados por município pelo Sistema de Informação de Agravos de Notificação. Os dados foram analisados com o auxílio do programa TerraView versão 4.2.2, por meio das bases cartográficas digitais dos municipais de Pernambuco e quantitativo populacional disponibilizado pelo Instituto Brasileiro de Geografia de Estatística.

Resultados: constatou-se a correlação linear positiva em relação às variáveis representadas pelas taxas brutas e ano de ocorrência e associação espacial entre os municípios.

Conclusão: o enfrentamento deste agravo requer mudanças de concepções culturais e suporte governamental para efetivação de açỗes intersetoriais de saúde.

DESCRITORES: Análise Espacial; Violência; Adolescente; Estupro; Saúde Pública.

\section{COMO REFERENCIAR ESTE ARTIGO:}

Abreu PD de, Santos ZC dos, Lúcio FP da S, Cunha TN da, Araújo EC de, Santos CB dos, et al. Análise espacial do estupro em adolescentes: características e impactos. Cogitare enferm. [Internet]. 2019 [acesso em "colocar data de acesso, dia, mês abreviado e ano"]; 24. Disponível em: http://dx.doi.org/10.5380/ce.v24i0.59743.

Este obra está licenciado com uma Licença Creative Commons Atribuição 4.0 Internacional.

${ }^{1}$ Enfermeira. Doutoranda em Enfermagem em Saúde Pública. Universidade de São Paulo. Ribeirão Preto, SP, Brasil. 9 ${ }^{2}$ Enfermeira. Mestre em Vigilância sobre Saúde. Docente de Enfermagem da Universidade Federal do Pernambuco. Vitória de Santo Antão, PE, Brasil. (

${ }^{3}$ Enfermeira. Mestre em Enfermagem. Universidade Federal de Pernambuco. Recife, PE, Brasil. $(2)$

${ }^{4}$ Físico. Mestre em Engenharia Biomédica. Bolsista Pesquisador da Fundação Oswaldo Cruz, Centro de Estudos da Saúde do Trabalhador e Ecologia Humana. Rio de Janeiro, RJ, Brasil. (2)

${ }^{5}$ Enfermeiro. Doutor em Enfermagem. Docente de Enfermagem da Universidade Federal de Pernambuco. Recife, PE, Brasil. (P)

${ }^{6}$ Matemática. Doutora em Estatística. Docente de Enfermagem da Universidade de São Paulo. Ribeirão Preto, SP, Brasil. $\bigcirc$ ${ }^{7}$ Enfermeira. Doutora em Enfermagem. Docente de Enfermagem da Universidade Federal de Pernambuco. Recife, PE, Brasil. () 


\title{
SPATIAL ANALYIS OF RAPE AGAINST ADOLESCENTS: CHARACTERISTICS AND IMPACTS
}

\begin{abstract}
Objective: To analyze the correlation between raw rates of rape and year of occurrence, and spatial patterns of notified cases of rape against adolescents.

Method: This was an ecological study, with a temporal trend, carried out based on notifications made between 2009 and 2014 of cases of rape against adolescents residing in the state of Pernambuco, Brazil, collected by the municipality through the Violent Crimes Notification Information System. The data were analyzed using TerraView software version 4.2.2. based on the municipal digital cartographic databases of Pernambuco and the population size provided by the Brazilian Institute of Geography and Statistics.

Results: There was a positive linear correlation between raw rates and year of occurrence and spatial association among municipalities.

Conclusion: Combating this violent crime requires changes in cultural conceptions and government support to effectively implement intersectoral health actions.
\end{abstract}

DESCRIPTORS: Spatial Analysis; Violence; Adolescent; Rape; Public Health.

\section{ANÁLISIS ESPACIAL DE LA VIOLACIÓN EN ADOLESCENTES: CARACTERISTICAS E IMPACTOS}

\begin{abstract}
RESUMEN:
Objetivo: Analizar la correlación entre tasas brutas de violencia por violación, año de ocurrencia y estándar espacial de ocurrencia de los casos notificados de violencia por violación contra adolescentes.

Método: Estudio ecológico con tendencia temporal, constituido por las notificaciones entre 2009 y 2014 referentes a casos de violencia por violación contra adolescentes residentes en Pernambuco, Brasil, divididos por municipio, recolectados del Sistema de Información de Enfermedades de Notificación. Datos analizados utilizando el programa TerraView versión 4.2.2, además de bases cartográficas digitales de los municipios de Pernambuco y cuantificación poblacional brindada por el Instituto Brasileño de Geografía y Estadística.

Resultados: Se constató correlación lineal positiva respecto de las variables representadas por las tasas brutas y año de ocurrencia, y asociación espacial entre los municipios.

Conclusión: Para enfrentar este estigma se requieren cambios en las concepciones culturales y soporte gubernamental que garantice la ejecución de acciones intersectoriales de salud.
\end{abstract}

DESCRIPTORES: Análisis Espacial; Violencia; Adolescente; Violación; Salud Pública. 
A violência configura-se em grave problema de saúde pública e resulta em danos biopsicossociais expressos a partir da dinâmica relacional societária. Nesta perspectiva, constitui-se em fenômeno complexo que precisa ser considerado dentro de determinado contexto, cultura e momento histórico em que acontece ${ }^{(1)}$. A Organização Mundial de Saúde (OMS) classifica a violência em três categorias de acordo com aquele que comete o ato: autoinfligida, interpessoal e coletiva, podendo ser de natureza física, sexual, psíquica, privações e negligência ${ }^{(2)}$.

A violência por estupro é considerada uma das formas mais agressivas de interação humana. Vários são os motivos para tal ato, contudo, basicamente envolve sentimentos de raiva, poder, crueldade erotizada e acasalamento oportunista ${ }^{(3)}$. Mundialmente, este agravo está intrinsicamente vinculado aos determinantes sociais e situações de vulnerabilidade que, agregados à perpetração das normatizações da sociedade, implícitas aos estereótipos, potencializa as relações de poder desiguais(4). As metas da ONU de enfrentamento à violência, combate ao crime e terrorismo estão inclusas na Agenda 30, entre os objetivos de desenvolvimento sustentável, com vistas ao provimento de sociedades pacíficas e livres do extremismo violento(5).

No Brasil, estima-se que 527 mil tentativas ou casos de estupros sejam consumados a cada ano, dos quais apenas $10 \%$ são reportados à polícia. De acordo com os dados do Sistema de Informação de Agravos de Notificação (SINAN), em 2011, foram notificados 12.087 casos de estupro no Brasil, destes casos $88,5 \%$ das vítimas eram do sexo feminino com predominância da faixa etária em menores de 13 anos de idade, de cor declarada preta ou parda por $51 \%$ dos indivíduos e com maior prevalência no grupo infanto-juvenil correspondendo a mais de $70 \%$ dos $\operatorname{casos}^{(6)}$.

O conhecimento da ocorrência e as formas de abordagem apropriadas para cada caso e o preparo de profissionais ligados às áreas da saúde, educação e afins, para a identificação (frequência e distribuição), notificação e mapeamento dos casos de estupro são de grande relevância. O geoprocessamento é uma importante ferramenta para o subsídio de planejamentos para a efetivação das políticas públicas integradas e intersetoriais, a fim de minimizar as inúmeras consequências decorrentes deste tipo de violência.

O mapeamento dos casos de estupro contra adolescentes em determinado espaço viabiliza a identificação da frequência, distribuição e a importância de diversos fatores que contribuem para as elevadas taxas deste evento. Diante do exposto, o presente estudo objetivou analisar a correlação entre as taxas brutas de violência por estupro e ano de ocorrência e o padrão espacial da ocorrência dos casos notificados de violência por estupro contra adolescentes.

\section{MÉTODO}

Estudo ecológico e de tendência temporal, efetuado no estado de Pernambuco (PE), no ano de 2018, a partir de dados secundários concernentes aos casos de violência por estupro provocado em adolescentes, no período de 2009 a 2014.

A população foi composta por 2.020 casos notificados de violência por estupro em adolescentes, dos 10 aos 19 anos de idade de acordo com a classificação da OMS (7), residentes no Estado de PE. Foram incluídos todos os dados registrados no intervalo temporal referido, disponibilizados pelo Departamento de Informática do Sistema Único de Saúde (DATASUS), a partir do Sistema de Informação de Agravos de Notificação (SINAN/ SVS/MS) ${ }^{(8)}$.

O estudo foi desenvolvido obedecendo três etapas: 1. Obtenção dos arquivos 
vetoriais para levantamento das bases cartográficas digitais, por malhas municipais relativas ao Estado de $\mathrm{PE}$, com subsequente produção das planilhas que correspondiam ao total da população na faixa etária de 10 a 19 anos idade de residentes no Estado de Pernambuco, no período estudado, disponibilizados pelo Instituto Brasileiro de Geografia e Estatística (IBGE); 2. Levantamento e elaboração das planilhas das notificações de violência por estupro contra adolescentes, residentes em PE, disponibilizadas pelo SINAN/SVS/MS (8); 3. Formatação, organização e correção ortográfica das planilhas.

A etapa analítica baseou-se no cálculo das taxas brutas de incidência de casos de violência por estupro contra adolescentes por município de residência e por ano de ocorrência, no período de 2009 a 2014. Os dados foram tabulados sob dois aspectos: expressão territorial e progressão temporal no período em estudo. A avaliação territorial subsidiou a associação espacial mediante os Índices de Moran Global e Local. Para suavizar os valores das taxas em consonância com os critérios estatísticos empíricos relacionados à influência exercida pela proximidade territorial, foi aplicada uma ferramenta bayesiana. $\mathrm{Na}$ análise de progressão temporal, foi avaliada a correlação linear entre a variável dependente representada pelas taxas brutas e a variável independente do ano de ocorrência.

Os dados foram analisados com o auxílio do programa TerraView, versão 4.2.2 para confecção dos Mapas Temáticos das taxas suavizadas pela técnica de Bayes Empírico e por associação espacial por meio do Índice de Moran (LisaMap)(9), a partir da variável dependente das taxas de violência por estupro, por suavização bayseana, em relação a 100 mil habitantes. A escolha desse tipo de análise deve-se à minimização de possíveis vieses decorrentes da instabilidade dos resultados por flutuações aleatórias decorrente das taxas brutas, visto que as estimativas em municípios com baixo quantitativo populacional tendem a revelar eventos por mero acaso ${ }^{(10)}$.

A análise inferiu, a priori, a construção dos mapas temáticos pelo estimador Bayes empírico global. Esse tipo de análise considera a média ponderada entre a taxa bruta local e taxa global de determinada região. A análise continuou com a elaboração dos mapas temáticos referentes às taxas bayesianas locais. Esse processo revela a estimativa local a partir das taxas da vizinhança geográfica da área a qual se deseja estimar ${ }^{(10)}$.

Posteriormente, foi avaliada a significância do Índice de Moran LisaMap - Local Indicator for Spatial Autocorrelation (Indicador Local de Auto correlação Espacial). Este indicador local, quando significativo, apresenta a correlação espacial entre os municípios vizinhos. Este tipo de análise produz a comparação dos valores locais, aleatorização e permutação das taxas dos municípios vizinhos e são categorizados em: maior que 0,05 indica significância baixa do índice obtido; menor que 0,05, a correlação é significativa, e suas as áreas classificadas em cinco diferentes níveis de significância: sem significância, significância de 0,05 (95\% de confiança), de 0,01 (99\% de confiança), de 0,001 (99,9\% de confiança), e de 0,0001 (99,99\% de confiança) $)^{(11)}$.

A escolha pela busca da significância do Índice de Moran local deu-se em decorrência da grande extensão do território estudado, assim, suscitaria em associações espaciais divergentes, sendo significativa a análise dos agrupamentos de áreas em relação à vizinhança ${ }^{(11)}$.

A visualização das taxas dos casos de violência por estupro procedeu com o modo para agrupamento das taxas: passos lguais. A subdivisão do valor mínimo e máximo em cinco fatias com intervalos iguais permitiu melhor interpretação das taxas nos mapas com referência à escala de cores ${ }^{(12)}$.

Por conseguinte, a análise exploratória dos dados obteve informações ocultas e identificação de comportamentos atípicos, a partir do coeficiente de correlação. $O$ coeficiente de determinação que delimita a força de associação linear, no qual um coeficiente de correlação zero indica a não existência de correlação entre as duas variáveis e o módulo do coeficiente de correlação igual a 1 indica uma correlação linear perfeita, estando assim distribuído em $\rho^{2}(0,1-0,3)$ fraco; $\rho^{2}(0,4-0,6)$ moderado; $\rho^{2}(0,7-1)$ forte ${ }^{(13)}$. 
Para a amostra deste estudo, foram utilizados dados secundários, disponíveis para acesso público no Sistema de Informação de Agravos de Notificação/DATASUS-TABNET e no IBGE. Esses sistemas reúnem informações relativas aos serviços de saúde, sistematizadas em planilhas, gráficos, mapas e textos para fins de pesquisa. Por se tratarem de dados de acesso público, foi dispensada a submissão ao Comitê de Ética em Pesquisa (CEP).

\section{RESULTADOS}

O presente estudo analisou a espacialização dos casos de violência por estupro produzida contra adolescentes, residentes no Estado de Pernambuco. A população foi composta por 2.020 casos notificados. O mapa temático relativo às taxas com suavização dos casos obtidos por meio do estimador Bayes Empírico Global para o período em estudo revelou em tons mais escuros os municípios com maiores taxas bayesianas globais, com destaque para o município de Petrolina com uma taxa de 89.68, conforme se apresenta na Figura 1.
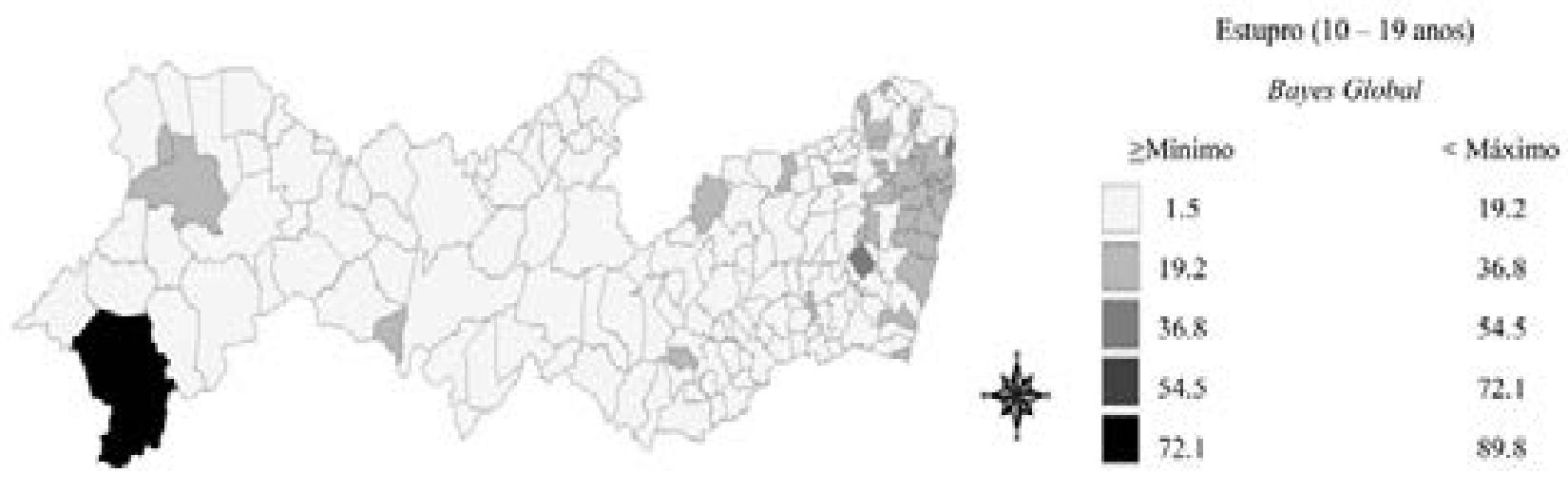

Figura 1 - Mapa Temático das taxas com suavização por Bayes Empírico Global, referente aos casos de violência por estupro de 10 a 19 anos, por 100 mil habitantes, nos municípios do Estado de Pernambuco. Recife, PE, Brasil, 2018

Na Figura 2 foi possível identificar as taxas bayesianas locais, que evidenciaram o município de Petrolina com taxa de 90.67.

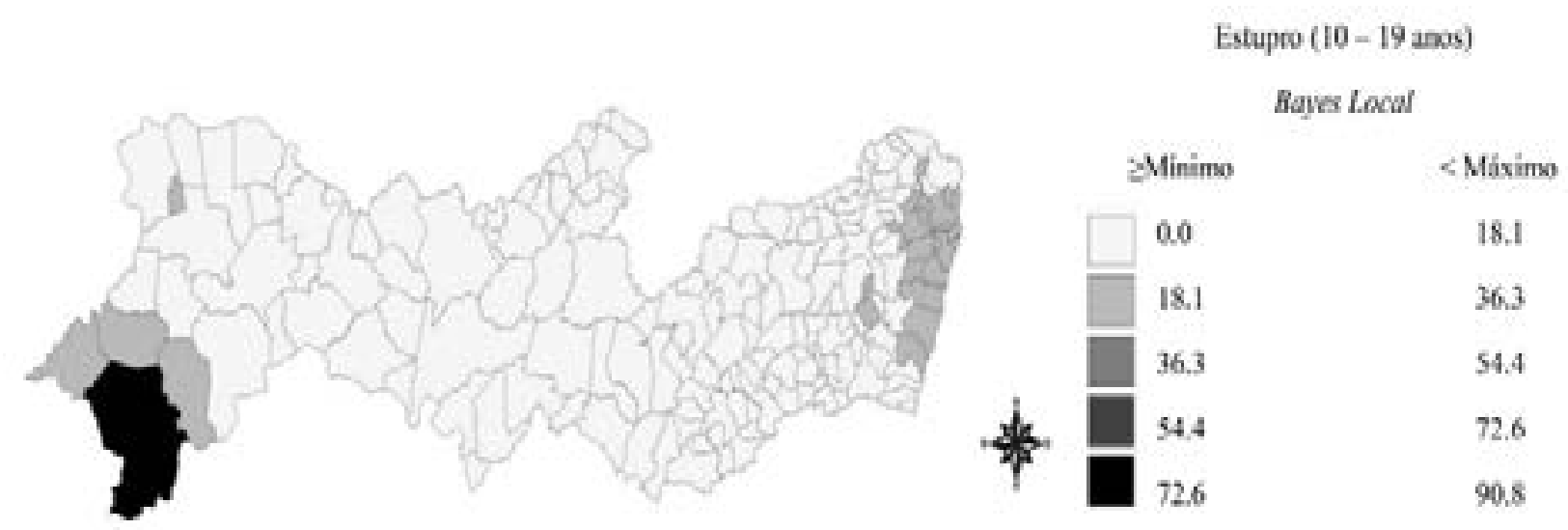

Figura 2 - Mapa Temático das taxas com suavização por Bayes Empírico Local, referente aos casos de violência por estupro em adolescentes de 10 a 19 anos, por 100 mil habitantes, nos município do Estado de Pernambuco. Recife, PE, Brasil, 2018 
A análise do Índice de Moran apresentou significância para faixa etária e agravo em estudo, obtendo p-valor igual a 0.002 e valor índice igual a 0.280864 .

A correlação linear entre as taxas brutas dos casos notificados de violência por estupro em adolescentes residentes em PE no período de 2009 a 2014 identificou correlação estatística positiva para ocorrência deste agravo e quanto à força de associação categorizada como forte, que possibilitou avançar na previsão da evolução das taxas até o ano de 2016, conforme se apresenta a seguir na Figura 3.

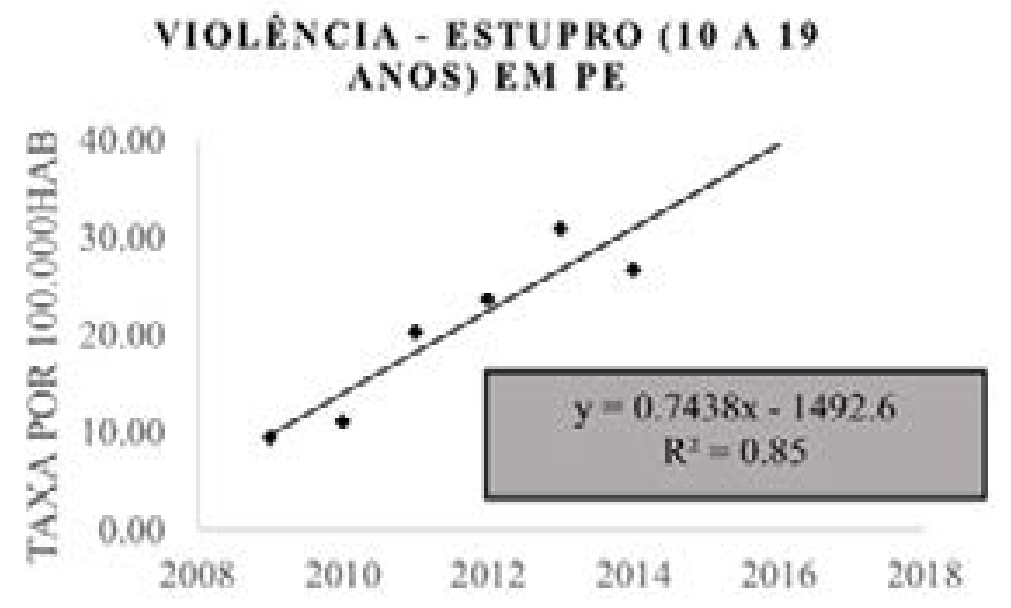

Figura 3 - Correlação Linear, no período de 2009 a 2014 e projeção até 2021 dos casos de violência por estupro em adolescentes, por 100 mil habitantes, residentes no Estado de Pernambuco. Recife, PE, Brasil, 2018

\section{DISCUSSÃO}

Os mapas temáticos por estimador bayes empírico global e local, para o período de 2009 a 2014, apontaram os municípios com maiores taxas de ocorrência dos casos de estupro entre os adolescentes. Evidenciou-se a dependência espacial dos casos, segundo índice de Moran, visto que os municípios vizinhos com elevadas/moderadas ou baixas taxas brutas de violência por estupro contra adolescentes apresentam mesmo padrão. $O$ perfil epidemiológico dos casos de violência por estupro contra adolescentes no Estado de PE desvelou que no grupo em estudo esse agravo foi mais evidente na mesorregião do São Francisco.

A correlação linear apresentou-se positiva para a ocorrência da violência por estupro contra adolescentes residentes em PE. A expressiva elevação do número de casos no decorrer dos anos mostrou tendência linear para este tipo de violência. Diante dessa constatação, é importante refletir sobre os determinantes sociais de saúde que influenciam o crescente aumento das taxas, a fim de construir estratégias intersetoriais que minimizem os impactos biopsicossociais provocados por esse tipo de agressão e que tem o potencial de repercutir em desajustes no decorrer da vida das vítimas.

A violência por estupro é um tipo violação que envolve o ato sexual impositivo, coercivo ou de abuso de poder para compelir a vítima sem que esta ofereça um consentimento válido. Os danos causados vão além da violação dos direitos humanos básicos, trazendo consigo profundo desequilíbrio do bem-estar físico, sexual, reprodutivo, emocional, mental e social da vítima acometida, o que pode resultar em significativa subestimação do nível real da ocorrência do agravo(14). 
O Brasil, país de grande extensão territorial, apresenta importantes desigualdades econômicas, sociais e culturais, desvela essa heterogeneidade, visto que o fenômeno acontece em todas as camadas sociais e independe da raça, classe, religião ou cultura ${ }^{(15)}$. Muitos são os grupos vulneráveis aos vários tipos de violência, dentre eles o estupro, todavia destaca-se a população infanto-juvenil, devido às características peculiares desta fase ${ }^{(16)}$.

No Brasil, o SINAN registrou na série histórica de 2009 a 2014 em torno de 64.080 casos de violência por estupro para a faixa etária de 10-19 anos ${ }^{(17)}$. Estudo realizado em Missouri (EUA) envolvendo 192 estudantes universitárias revelou que uma em cada 20 estudantes universitárias relatam já terem vivenciado a experiência de estupro, destas $41,5 \%$ divulgou a violação por estupro, indicando o impacto substancial da violação nesta configuração. Mais de dois terços das vítimas entrevistadas informaram que contaram a pelo menos uma pessoa sobre a violência, contudo, na maioria das vezes as fontes são de apoio informal, como amigos e familiares ${ }^{(18)}$.

A região Nordeste do Brasil apresenta 14.873casos registrados de violência por estupro, com predomínio do número de registros para o sexo feminino, com percentual de $74 \%$ das vítimas. Estas estimativas traduzem em cifras o segundo lugar no ranking nacional de casos que envolvem este fenômeno(17).

O estado de Pernambuco possui comportamento epidemiológico semelhante em nível internacional e nacional, ocupando o segundo lugar entre os estados da região Nordeste do país, com percentual mais elevado de casos de violência por estupro, tendo o sexo feminino com 3.215 (85\%) do total de casos registrados ${ }^{(17)}$.

No presente estudo observa-se a marcante presença do fenômeno em dois territórios extremos do estado de Pernambuco: Região Metropolitana do Recife e Mesorregião do São Francisco, esta última com uma população estimada de 478.117 habitantes ${ }^{(19)}$. Nesta última mesorregião encontra-se o município de Petrolina, que apresenta grande destaque em relação aos demais municípios da região.

As características econômicas, demográficas e geográficas deste município possuem importantes áreas de desenvolvimento industrial e de comércio, interligados por rodovias e por linha aérea, podendo ser uma das explicações para o fenômeno. Ressalta-se que este polo é atrativo para uma população migratória em busca de melhores oportunidades de emprego. Outra possibilidade é que a rede de acolhimento aos casos de estupro está mais bem aparelhada nesta área e, portanto, consegue captar maior número de denúncias e de notificações.

O elevado índice de casos de estupro em determinados municípios pernambucanos, com destaque para Petrolina como já mencionado, tem como perfil de vítimas pessoas do sexo feminino (98,7\%), possuem raça/cor parda (35\%). Quanto ao perfil do agressor, $24 \%$ dos casos possuíam a notificação do agressor, com destaque: namorado (10,5\%), desconhecido $(8 \%)$ e padrasto $(4,5 \%)^{(17)}$.

Estes achados sugerem que este tipo de violação contra mulheres pode estar relacionado à cultura do estupro, a qual perpetua a hierarquia do poder sexual masculino sobre o feminino(20). Estudos têm apresentado perfis semelhantes quanto às vítimas cuja maioria é do sexo feminino, com faixa etária predominante em menores de 13 anos, e cor de pele parda e preta. Em relação ao perfil do agressor, os mesmos estudos apontam preponderância para o padrasto e um deles destaca este agressor como usuário de álcool e outras drogas ${ }^{(6,21)}$.

Os achados do presente estudo corroboram com a predominância deste tipo de violência no sexo feminino(22), provavelmente pela histórica condição da mulher na sociedade que vivencia com certa complacência as diversas formas de violência relacionada à persistência do sistema patriarcal(23). Tal sistema é perpetuado, e intrínseco à cultura masculina independentemente da idade do agressor ${ }^{(21)}$. 
Quando o estupro tem como vítima os meninos, observa-se a maior demanda desses casos para os serviços de média e alta complexidade de saúde, para o atendimento emergencial, em detrimento dos serviços especializados no acolhimento e suporte social e judiciário(22). Por outro lado, não se pode menosprezar as altas proporções de crianças do gênero masculino vítimas de estupro, principalmente a partir dos seis anos de idade. A subnotificação nestes casos possivelmente refere-se a fatores culturais, preconceitos e estigmas relacionados à sexualidade masculina ${ }^{(23)}$.

Estudo realizado nos EUA associou o uso da mídia sexual (filmes, músicas, televisão e jogos) ao aumento das práticas de violência sexual. A representação das práticas sexuais rege a normalização, difundida por meios de comunicação, em que todos os casais devem realizar sexo, o que corrobora com o cenário de pressão exercido ao parceiro a ter relações sexuais, sendo emergente a investigação dos impactos midiáticos à juventude ${ }^{(24)}$.

É necessária a utilização de recursos e espaços territoriais para a elaboração de ações educativas com debates acerca do patriarcalismo, igualdade de gênero, sexo e enfrentamento da cultura do estupro. Além disso, a responsabilização social em encorajar as denúncias, acolhimento e proteção às vítimas são processos fundamentais para desmitificar o sentimento de culpa e proporcionar alternativas para a prevenção e superação(25).

O enfermeiro destaca-se como educador em saúde nas atividades de promoção da saúde mediante a Política Nacional de Atenção Básica (PNAB), para a construção de estratégias com os Núcleos de Apoio nas dimensões clínico-assistencial e técnicopedagógica, em ações do Programa Saúde na Escola (PSE) para o cuidado de rede no território delimitado segundo a área de abrangência da Estratégia Saúde da Família.

A escola é um espaço privilegiado para a atuação do profissional de enfermagem, visto que esse pode viabilizar o encontro da educação e saúde, no enfrentamento às vulnerabilidades que permeiam o pleno desenvolvimento de crianças e adolescentes na Educação. No âmbito do cuidado da Enfermagem, devem-se considerar os recursos do território para o enfrentamento da violência, sobretudo entre as pessoas em situação de vulnerabilidade social.

O presente estudo apresentou como limitação a possível subnotificação dos casos de violência por estupro em adolescentes residentes em PE. A operacionalização dos Sistemas de Informação em Saúde por diferentes unidades municipais pode divergir e ocasionar perda de informações. A produção dos dados irá depender desde a identificação dos casos suspeitos pelos serviços de saúde, ao diagnóstico, registro e organização dos dados digitalizados, etapas essenciais para a condução epidemiológica consistente desse agravo.

A contribuição deste estudo consiste na identificação das áreas com maior quantitativo dos casos de violência por estupro na população adolescente, a fim de subsidiar o enfrentamento desse agravo no âmbito comunitário. Diante disso, este estudo será o ponto de partida para outras pesquisas que fundamentem o conhecimento acerca dos fatores que corroboram para a opressão social dos adolescentes às práticas de violência sexual.

A visão do agravo no Estado a partir do Geoprocessamento configura-se em importante ferramenta epidemiológica para o reconhecimento das áreas de risco no território, possibilitando a aplicação de conjunto de técnicas que contribuam para o desenvolvimento e fortalecimento de ações em Saúde Coletiva. Neste cenário, a vulnerabilidade dos adolescentes ao sexo coercivo, evidente em determinadas regiões de PE, desvela a emergente necessidade de atuação dos gestores sociais, de saúde e sociedade civil para o enfrentamento da violência por estupro.

O protagonismo da Enfermagem no enfrentamento às vulnerabilidades demanda 
compromisso de cidadania. O provimento da prevenção à violência sexual requer a participação social na desmistificação cultural do estupro na sociedade. Neste sentido, o enfrentamento demanda do conhecimento territorial para a utilização de recursos disponíveis na comunidade e construção de estratégias de educação em saúde, a fim de propiciar o cuidado integral aos adolescentes.

\section{AGRADECIMENTOS}

19).

O presente estudo foi financiado pelo PROCAD (CAPES n: 23.038.000.984.2014-

\section{REFERÊNCIAS}

1. Garbin CAS, Gomes AMP, Gatto RCJ, Garbin AJI. Um estudo transversal sobre cinco anos de denúncia sobre violência contra crianças e adolescentes em Araçatuba - São Paulo. J Health Sci [Internet]. 2016 [acesso em 15 set 2017]; 18(4). Disponível em: http://revista.pgsskroton.com.br/index.php/JHealthSci/ article/view/3293.

2. Organização Mundial de Saúde (OMS). Informe mundial sobre la violencia y la salud. [Internet] Washington, DC: OMS; 2014 [acesso em 15 set 2017]. Disponível em: http://dx.doi.org/10.1590/S003646652003000300014.

3. Miller L. Rape: sex crime, act of violence, or naturalistic adaptation? Aggression and Violent Behavior. [Internet]. 2014 [acesso em 15 set 2017]; 19(1). Disponível em: http://dx.doi.org/10.1016/j. avb.2013.11.004.

4. United Nations Educational, Scientific and Cultural Organization (UNESCO). School Violence and Bullying: Global Status Report. Seoul, Republic of Korea: UNESCO; 2017.

5. Organização das Nações Unidas. Objetivos de Desenvolvimento Sustentável. [Internet]. 2017 [acesso em 25 jun 2017]. Disponível em: https://nacoesunidas.org/pos2015/agenda2030/.

6. Cerqueira D, Coelho DSC. Estupro no Brasil: uma radiografia segundo os dados da Saúde (versão preliminar). Brasília: IPEA; 2014. (Nota técnica n. 11).

7. Organização Mundial de Saúde (OMS). Organização Pan-Americana da Saúde. Relatório Mundial sobre violência e saúde. [Internet]. Genebra: OSM; 2002 [acesso em 15 set 2017]. Disponível em: https://www. opas.org.br/wp-content/uploads/2015/09/relatorio-mundial-violencia-saude.pdf.

8. Departamento de Informática do SUS (DATASUS). Doenças e Agravos - Violência Interpessoal/ Autoprovocada. [Internet]. 2017 [acesso em 25 jun 2017]. Disponível em: http://tabnet.datasus.gov.br/cgi/ tabcgi.exe?sinannet/cnv/violebr.def.

9. Instituto Nacional de Pesquisas Espaciais (INPE): Programa TERRAVIEW 4.2.2. [Internet]. 2010 [acesso em 15 set 2017]. Disponível em: http://www.dpi.inpe.br/terraview/index.php.

10. Honorato T, Lapa PPA, Sales CMM, Reis-Santos B, Tristão-Sá R, Bertolde Al et al. Análise espacial do risco de dengue no Espírito Santo, Brasil, 2010: uso de modelagem completamente Bayesiana. Rev. bras. epidemiol. [Internet]. 2014 [acesso em 15 set 2014]; 17(Suppl 2). Disponível em: https://doi. org/10.1590/1809-4503201400060013.

11. Nunes FG. Análise exploratória espacial de indicadores de desenvolvimento socioambiental das regiões de planejamento do norte e nordeste goiano. Ateliê Geográfico. [Internet]. 2013 [acesso em 15 set 2017]; 7(1). Disponível em: https://doi.org/10.5216/ag.v7i1.19809. 
12. Francisco CN. Conceitos de Geoprocessamento: Sistemas de Informações Geográficas. 2. ed. Niterói (RJ): Universidade Federal Fluminense; 2014.

13. Mukaka MM. Statistics Corner: A guide to appropriate use of correlation coeficiente in medical research. Malawi Med J. [Internet]. 2012 [acesso em 15 set 2017]; 24(30). Disponível em: https://www. ncbi.nlm.nih.gov/pmc/articles/PMC3576830/.

14. Organização Mundial de Saúde (OMS). Relatório Mundial Sobre a Prevenção da Violência. São Paulo: Núcleo de Estudos da Violência da Universidade de São Paulo, Organização Mundial de Saúde; 2015. 17p. Relatório final.

15. Rodrigues JL, Lima AP, Nagata JY, Rigo L, Cericato GO, Franco A, et al. Domestic violence against children detected and managed in the routine of dentistry: a systematic review. J Forensic Leg Med. [Internet]. 2016 [acesso em 15 set 2017]; 43. Disponível em: https://doi.org/10.1016/j.jflm.2016.07.006.

16. Nunes AJ, Sales MCV. Violência contra crianças no cenário brasileiro. Cienc. saude colet. [Internet]. 2016 [acesso em 15 set 2017]; 21(3). Disponível em: https://doi.org/10.1590/141381232015213.08182014.

17. Departamento de Informática do SUS (DATASUS). Doenças e Agravos - Violência Interpessoal/ Autoprovocada. [Internet]. 2015 [acesso em 15 set 2017]. Disponível em: http://tabnet.datasus.gov.br/cgi/ tabcgi.exe?sinannet/cnv/violebr.def.

18. Paul LA, Kehn A, Gray MJ, Salapska-Gelleri J. Perceptions of, and Assistance Provided to, a Hypothetical Rape Victim: Differences Between Rape Disclosure Recipients and Nonrecipients. J Am Coll Health [Internet]. 2014 [acesso em 15 set 2017]; 62(6). Disponível em: https://doi.org/10.1080/07448481.2 014.917651.

19. Instituto Brasileiro de Geografia e Estatística (IBGE). Estimativa Populacional 2013. [Internet]. 2017 [acesso em 15 set 2017]. Disponível: http://www.ibge.gov.br/home/estatistica/populacao/estimativa2013/ default.shtm.

20. Strain ML, Hockett JM, Saucier DA. Precursors to Rape: Pressuring Behaviors and Rape Proclivity. Springer Publishing Company Connect. [Internet]. 2015 [acesso em 15 set 2017]; 30(2). Disponível em: https://doi.org/10.1891/0886-6708.VV-D-13-00051.

21. Pinto Júnior AA, Lopes DC, Pinheiro VS, Ortiz MM, Oliveira SL. Perfil da Violência Sexual Doméstica Contra Crianças e Adolescentes no Município de Dourados. Rev. Psicol Saude. [Internet]. 2012 [acesso em 15 set 2017]; 4(1). Disponível em: http://dx.doi.org/10.1590/1413-81232018235.18662016.

22. Hohendorff JV, Habigzang LF, Koller SH. Violência sexual contra meninos: dados epidemiológicos e consequências. Psicol. USP. [Internet]. 2012 [acesso em 15 set 2017]; 23(2). Disponível em: http://dx.doi. org/10.1590/S0103-65642012005000007.

23. Oliveira JR de, Costa MCO, Amaral MTR, Santos CA, Assis SG de, Nascimento OC. Violência sexual e coocorrências em crianças e adolescentes: estudo das incidências ao logo de uma década. Ciênc. saúde coletiva. [Internet]. 2014 [acesso em 15 set 2017]; 19(3). Disponível em: http://dx.doi.org/10.1590/141381232014193.18332013.

24. Ybarra ML, Strasburger VC, Mitchell KJ. Sexual Media Exposure, Sexual Behavior, and Sexual Violence Victimization in Adolescence. Clin Pediatr. [Internet]. 2014 [acesso em 15 set 2017]; 53(13). Disponível em: http://dx.doi.org/10.1177/0009922814538700.

25. Barnett MD, Sligar KB, Wang CDC. Religious Affiliation, Religiosity, Gender, and Rape Myth Acceptance: Feminist Theory and Rape Culture. J Interpers Violence [Internet]. 2018 [acesso em 15 set 2017]; 33(8). Disponível em: http://dx.doi.org/10.1177/0886260516665110. 
Recebido: 01/06/2018

Finalizado: 24/06/2019

Autor Correspondente:

Paula Daniella de Abreu

Universidade de São Paulo

Av. dos Bandeirantes, 3900 - 14040-902 - Ribeirão Preto, SP, Brasil

E-mail: pauladdabreu@gmail.com

Contribuição dos autores:

Contribuições substanciais para a concepção ou desenho do estudo; ou a aquisição, análise ou interpretação de dados do estudo - PDA, ZCS, FPSL, TNC, ECA, CBS, EMRV

Elaboração e revisão crítica do conteúdo intelectual do estudo - PDA, ZCS, FPSL, TNC, ECA, CBS, EMRV

Aprovação da versão final do estudo a ser publicado - PDA, ZCS, FPSL, TNC, ECA, CBS, EMRV

Responsável por todos os aspectos do estudo, assegurando as questões de precisão ou integridade de qualquer parte do estudo - PDA, ZCS, FPSL, TNC, ECA, CBS, EMRV 